\title{
The geometry of quantum codes
}

\author{
Jürgen Bierbrauer Giorgio Faina Massimo Giulietti \\ Stefano Marcugini Fernanda Pambianco
}

full screen

close

quit

\begin{abstract}
We give a geometric interpretation of additive quantum stabilizer codes in terms of sets of lines in binary symplectic space. It is used to obtain synthetic geometric constructions and non-existence results. In particular several open problems are removed from Grassl's database [13].
\end{abstract}

Keywords: quantum codes, symplectic geometry, Hermitian form, additive codes, Blokhuis-Brouwer construction, APN function, spread, quantum cap

MSC 2000: 51E21, 94B05, 94B27, 94B60

\section{Introduction}

The most popular construction of quantum error-correcting codes was developed in [6]. It uses a special class of additive quaternary codes. Those codes are known as quantum stabilizer codes. We start in Section 2 by recalling basic facts concerning quaternary additive codes and their geometric representation as developed in [5, 3, 4]. Quantum stabilizer codes live in symplectic geometry. Starting point for our investigation is a geometric interpretation of quantum stabilizer codes in terms of systems of lines in symplectic geometry. It is one of the aims to obtain more transparent constructions and bounds using geometric terminology. The paper is mostly concerned with codes of distance $d \leq 4$. Our simplified approach allows us to derive more general constructions. In particular we resolve a number of existence questions (see Grassl's database [13]). Among the results are constructions of new pure codes

$$
\text { [[36, 29, 3]], [[37, 30, 3]], [[38, 31, 3]], [[81, 73, 3]], [[756, 740, 4]], [[5040, 5020, 4]] }
$$

and proofs of nonexistence for parameters [[39, 32, 3]], [[82,74, 3]], [[83, 75, 3]]. For the geometric approach to linear codes see [1]. The general geometric description of quantum stabilizer codes is given in Section 2. Section 3 discusses 


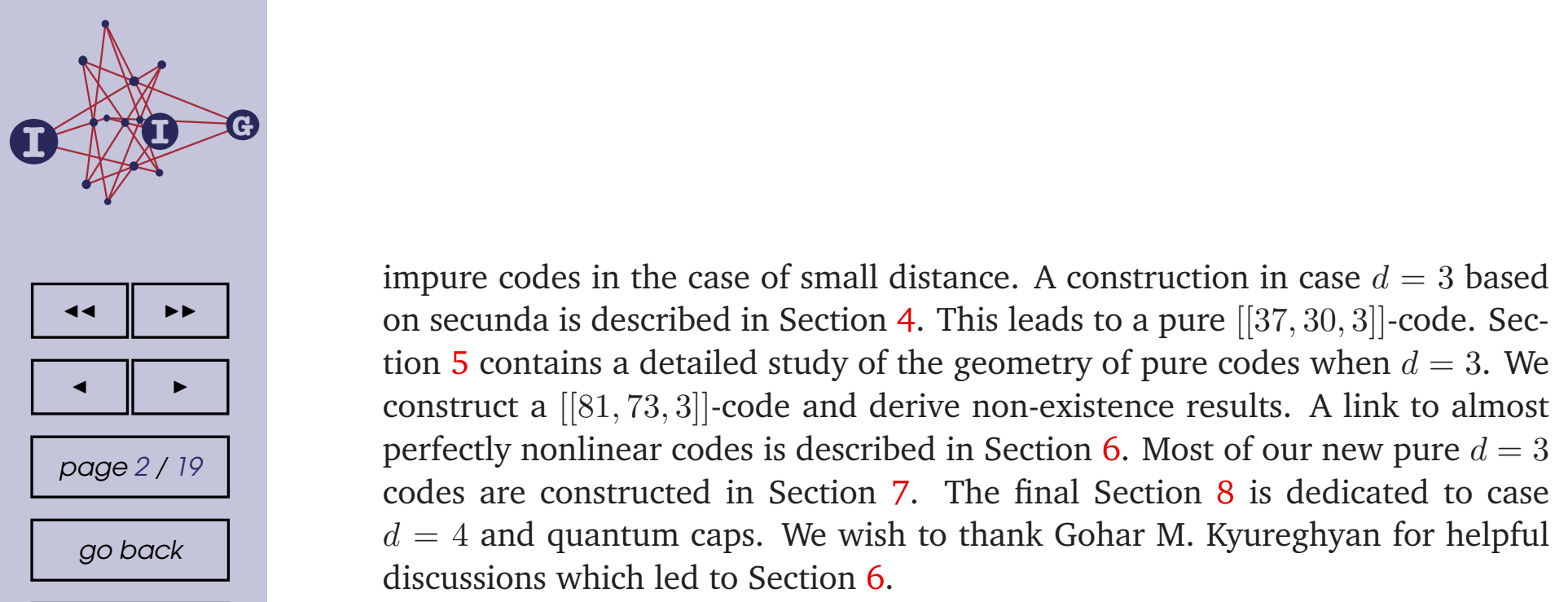

full screen

close

quit

ACADEMIA

PRESS

N

$\underline{\underline{\underline{\Pi \|}}}$

UNIVERSITEIT

GENT

\section{Geometric description of quantum stabilizer codes}

We use the notion of an additive code as the relaxation of the notion of a linear code, where the alphabet is not considered as a finite field but only as a vector space over some ground field. Here we consider only the quaternary case, where the ground field is $\mathbb{F}_{2}$.

Definition 2.1. Let $k$ be such that $2 k$ is a positive integer. An additive quaternary $[n, k]_{4}$-code $\mathcal{C}$ (length $n$, dimension $k$ ) is a $2 k$-dimensional subspace of $\mathbb{F}_{2}^{2 n}$, where the coordinates come in pairs of two. We view the codewords as $n$-tuples where the coordinate entries are elements of $\mathbb{F}_{2}^{2}$.

A generator matrix of $\mathcal{C}$ is a binary $(2 k, 2 n)$-matrix whose rows form a basis of the binary vector space $\mathcal{C}$.

In the case of quantum stabilizer codes we view the ambient space $\mathbb{F}_{2}^{2 n}$ as a binary symplectic space, where each of the $n$ parameter sections corresponds to a hyperbolic plane, equivalently a 2-dimensional symplectic space. Each codeword is therefore a vector in the $2 n$-dimensional symplectic geometry over $\mathbb{F}_{2}$.

Definition 2.2. A quaternary quantum stabilizer code is an additive quaternary code $C$ which is contained in its dual, where duality is with respect to the symplectic form.

Describe $C$ by a generator matrix $M$. Each of the $n$ coordinate sections contains 2 columns which we view as points in binary projective space. The geometric description of the quantum code is in terms of the system of $n$ lines (the codelines) generated by those $n$ pairs of points. This terminology is not always fully justified. It is possible that 0-columns occur and that the two points in a coordinate section are identical. These degenerate cases are best described by the strength:

Definition 2.3. Let $C$ be a quaternary additive code of length $n$, with generator matrix $M$. The strength of $C$ is the largest number $t$ such that any $t$ codelines are in general position. 
For example $t \geq 1$ means that each coordinate section does indeed generate a codeline, and $t \geq 2$ means that those $n$ codelines are pairwise skew. The general definition of the parameters of a quantum stabilizer code is as follows.

Definition 2.4. An $[[n, m, d]]$-code $C$ where $m>0$ is a quaternary quantum stabilizer code of binary dimension $n-m$ satisfying the following: any codeword of $C^{\perp}$ having weight at most $d-1$ is in $C$.

The code is pure if $C^{\perp}$ does not contain codewords of weight $\leq d-1$, equivalently if $C$ has strength $t \geq d-1$.

An $[[n, 0, d]]$-code $C$ is a self-dual quaternary quantum stabilizer code of strength $t=d-1$.

In particular, whenever some $\leq d-1$ codelines are not in general position there is a hyperplane containing all the other codelines. We prefer working with pure quantum codes. The reason why in Definition 2.4 we did not distinguish between codelines and codepoints is that codepoints are easily disposed of:

Lemma 2.5. Assume there is an $[[n, m, d]]$-code, $d \geq 2$, one of whose codeobjects is not a line but a point (equivalently, the code does not have strength $\geq 1$ ). Then the remaining objects define an $[[n-1, m, d]]$-code. Conversely, if an $[[n-1, m, d]]$-code exists, then there is an $[[n, m, d]]$-code one of whose codeobjects is a point.

Proof. Let a point $P=L_{n}$ be a codeobject of an $[[n, m, d]]$-code $C$. By the definition of the symplectic product there is a nonzero codeword in $C^{\perp}$ with entries 00 in all but the last coordinate section. By Definition 2.4 this codeword is in $C$. Write it as last row of a generator matrix. Consider the matrix obtained by removing the last row and $L_{n}=P$. This is again a self-dual code and it generates an $[[n-1, m, d]]$-quantum code.

If a generator matrix of an $[[n-1, m, d]]$-code is given we can add an extra coordinate consisting of a 0-column and a 1-column, and an extra row with all entries 00 except for the additional column. This defines an $[[n, m, d]]$-code whose $n$-th codeobject is a point.

As $[[n-1, m, d]]$-code implies an $[[n, m, d]]$-code we can think of the basic problem as minimizing $n$ when $m, d$ are given. Lemma 2.5 shows that we can assume all codeobjects to be lines. The role of codepoints is restricted to the construction of an $[[n, m, d]]$-code from an $[[n-1, m, d]]$-code.

Consider a pure $[[n, m, d]]$-code. In our quaternary notation it is the dual of an additive $[n,(n+m) / 2, d]_{4}$-code. Most important is the self-orthogonality condition. Imagine a generator matrix of $C$. The codewords are linear combinations of rows. Those factors describe a hyperplane in $\operatorname{PG}(n-m-1,2)$. Consider two codewords of $C$ and the hyperplanes $H_{1}$ and $H_{2}$ corresponding 

Theorem 2.8. The following are equivalent:

- a pure quantum code $[[n, n-2 m, t+1]]$ which is linear over $\mathbb{F}_{4}$;

- a set of $n$ points in $\mathrm{PG}(m-1,4)$ all $t$ of which are in general position and such that the intersection size with any hyperplane has the same parity as $n$;

- an $[n, m]_{4}$ code of strength $t$ all of whose weights are even;

go back

full screen

close

- an $[n, m]_{4}$ code of strength $t$ which is self-orthogonal with respect to the Hermitian form.

Proof. Consider the equivalence of the first two items, using Theorem 2.6. Observe that a hyperplane of $\operatorname{PG}(m-1,4)$ is a secundum of $\operatorname{PG}(2 m-1,2)$. This shows that the first item implies the second. Assume now the condition of the second item is satisfied and consider a secundum $S$ which is not an $\mathbb{F}_{4}$-hyperplane. Let $K=S \cap \omega S$ be the largest $\mathbb{F}_{4}$-subspace contained in $S$. Then $K$ has binary codimension 4 in $V=\mathbb{F}_{2}^{2 m}$. The quaternary hyperplanes containing $K$ form a spread $\left\{S_{1}, \ldots S_{5}\right\}$ in the factor space $V / K$ and $S / K$ is a line in this factor space $\mathrm{PG}(3,2)$. Let $m$ be the number of codepoints (lines if considered binary) contained in $K$ and $a_{i}$ the number of codepoints in $S_{i}$ but not in $K$. Then $n=m=\sum a_{i}$ is the number of codepoints, and the quaternary condition applied to $S_{i}$ shows $\sum_{j \neq i} a_{j}$ even. It follows that all $a_{i}$ have the same parity. The number of codepoints disjoint from $S$ is the sum of two of those numbers and therefore even.

The equivalence with the third item is obvious. Finally, the Hermitian form is identically zero if and only if each vector is orthogonal to itself. In the quaternary case this is equivalent with the weights being even.

\section{The role of impurity}

Proposition 3.1. An $[[n, m, d]]$ of strength 0 is equivalent with an $[[n-1, m, d]]$. An $[[n, m, d]]$ with a double codeline is equivalent with an $[[n-2, m, d]]$.

Proof. The first statement is a special case of Lemma 2.5. The same observation yields the second statement.

Theorem 3.2. Let $C$ be an $[[n, n-r, d]]$-code of strength $\geq 1$ without double codelines, where $d \geq 3$. Let $P_{1}, \ldots, P_{k}$ be the points on more than one codeline, denote by $u_{i}$ the valency of $P_{i}$. Then

$$
n \leq\left(\sum u_{i}\right)+\left(2^{r-\sum\left(u_{i}-1\right)}-1-k\right) / 3 .
$$





\section{A quadratic construction}

Not all examples are of this form. For example, a pure $[[9,3,3]]$ can also be constructed via the elliptic quadric in $\mathrm{PG}(5,2)$. It has 27 points and there is a spread partitioning those 27 isotropic points. Each secundum meets it in odd cardinality.

More generally consider the hyperbolic and elliptic quadrics $Q^{+}(2 m-1)$ and $Q^{-}(2 m-1)$ in $\mathrm{PG}(2 m-1,2)$. Their number of points is $\left(2^{m}-1\right)\left(2^{m-1}+1\right)$ in the hyperbolic, $\left(2^{m}+1\right)\left(2^{m-1}-1\right)$ in the elliptic case. Let $m=2 l$ be even in the hyperbolic case, $m=2 l+1$ odd in the elliptic case. The quadrics can be partitioned into generators. Those generators, of projective dimension $2 l-1$, can themselves be partitioned into lines. It follows that the quadrics can be partitioned into lines. As each quadric in binary projective space is either empty or has an odd number of isotropic points, it follows that the secundum condition is satisfied in those cases. We have proved the following result.

Theorem 5.2. Let $n=\left(2^{2 l-1}+1\right)\left(4^{l}-1\right) / 3$ for $l \geq 2$. Then there is a pure

$$
\text { [[n, } n-4 l, 3]] \text {-code. }
$$

Let $n=\left(2^{2 l+1}+1\right)\left(4^{l}-1\right) / 3$ for $l \geq 1$. Then there is a pure

$$
[[n, n-4 l-2,3]] \text {-code. }
$$

Examples are parameters $[[9,3,3]],[[45,37,3]],[[155,145,3]]$.

\section{Using the complement}

When $n$ is large it is natural to consider the set of points that are not contained in the union of the codelines.

Theorem 5.3. Let $n, r$ be given and $y=2^{r}-1-3 n$. A pure $[[n, n-r, 3]]$ quantum code is equivalently described by the following:

- a self-orthogonal projective code $C$ of length $y$ and dimension $\leq r$;

- a partial spread of $n$ lines in $\mathrm{PG}(r-1,2)$ covering the points which are not columns of a generator matrix of $C$.

Proof. Start from a pure quantum code, let $Y$ be the set of $y$ points in PG $(r-1,2)$ not contained in a line of the corresponding partial spread and $X$ its complement. Then $y$ and $n$ have different parities. Let $S$ be a secundum. Then $S \cap X$ has the same parity as $n$, so $S \cap Y$ has the parity of $y$. 
Let $G$ be the $(r, y)$-matrix whose columns are the points of $Y$, and $C$ the projective code generated by $G$. We saw that each secundum $S$ meets $Y$ in the parity of $y$. Let $H$ be a hyperplane, $m=|H \cap Y|$. Then all weights of the corresponding code have the same parity. This is therefore even. It follows that $m$ has the same parity as $y$ and all words of $C$ have even weight. Let $u, v \in C$ and denote by $a, b, c, d$ the number of coordinates where the entries of $u$ and $v$ are $(0,0),(0,1),(1,0),(1,1)$, respectively. We saw that $a$ has the parity of $y$ whereas $b, c$ are even. As $a+b+c+d=y$ this implies that $d$ is even and $u \cdot v=0$. We have that $C$ is a self-orthogonal projective code. Clearly we have reached an equivalent description.

In Theorem 5.3 it is impossible that $y$ is $2 \bmod 3$ (otherwise $2^{r}$ would have to be a multiple of 3), and $y$ is a multiple of 3 if $m$ is even, $y$ is $1 \bmod 3$ if $m$ is odd. This description in terms of the complement $Y$ is particularly useful when $y=2^{r}-1-3 n$ is small.

Definition 5.4. Let $\mathcal{Y}$ be the family of point sets $Y \subseteq \mathrm{PG}(l, 2)$ with the property that the matrix with the elements of $Y$ as columns generates a self-orthogonal code. Let $y=|Y|$ be the length and $k$ the dimension of this code.

Observe that subspaces $\operatorname{PG}(k, 2)$ for $k \geq 2$ belong to $\mathcal{Y}$ and that $\mathcal{Y}$ is closed under symmetric sums.

Proposition 5.5. Let $Y \in \mathcal{Y}$. If $y \leq 13$, then one of the following occurs.

(1) $y=7, k=3$ and $Y$ a plane;

(2) $y=8, k=4$ and $Y$ is the complement of a plane. The corresponding code is the $[8,4,4]_{2}$ extended Hamming code;

(3) $y=11, k=5$ and $Y=E_{1} \oplus E_{2} \oplus S$ is the symmetric sum of two planes $E_{1}, E_{2}$ meeting in a point and a solid containing $E_{2}$;

(4) $y=12, k=5$ and $Y$ is the exclusive or $E_{1} \oplus E_{2}$ of two planes meeting in a point;

(5) $y=12, k=6$ and $Y$ is equivalent to the set of 6 -tuples of weights 1 or 5 ;

(6) $y=13, k=6$ and $Y=E_{1} \oplus E_{2} \oplus S$ is the symmetric sum of two skew planes $E_{1}, E_{2}$ and a solid containing $E_{2}$.

Proof. Obviously $y \leq 6$ is impossible, and $Y$ is a plane if $y=7$. If $y=8$ then $k=4$. We must have the complement of a plane. Considering complements cases $y=9$ and $y=10, k=4$ are excluded.

Let now $y=10, k=5$ and write a generator matrix $(I \mid P)$. Then $P$ is a quadratic matrix with rows of odd weight and even pairwise intersections. No 




This construction is not very useful in the situation when $Y \subset H$, where $H$ is a hyperplane of $V_{m}$. The reason is that the remaining spread lines together with $Y$ would fill a $V_{m-2}$. This would describe a quantum code obtained by the recursive construction of Corollary 4.3. It is therefore desirable to have more constructions of sum-disjoint partitions. We know that bijective APN-functions are examples. Unfortunately no bijective APN-functions on $\mathrm{GF}\left(2^{n}\right)$ are known when $n$ is even.

\section{Elementary moves}

Here is what seems to be the most elementary way of constructing a sumdisjoint partition from another one. Let $\Sigma=S(\pi)$ be given and $\left\{x, x^{\prime}\right\},\left\{y, y^{\prime}\right\} \in$ $\pi$ (so that $x+x^{\prime}, y+y^{\prime} \in \Sigma$ ). Assume $x+y, x^{\prime}+y^{\prime} \notin \Sigma$. Then replacing the two pairs above by $\{x, y\},\left\{x^{\prime}, y^{\prime}\right\}$ we obtain another sum-disjoint partition whose set of sums $\Sigma^{\prime}$ differs from $\Sigma$ in that $x+x^{\prime}, y+y^{\prime}$ have been removed and $x+y, x^{\prime}+y^{\prime}$ added.

\section{Constructions}

In this section we will construct pure quantum codes $[[17,11,3]],[36,29,3]]$ and $[[38,31,3]]$. The latter two parameters are new. For the first two the construction is direct and easy to verify. In length 38 we start from the BB-construction (Proposition 6.6) which is modified by three elementary moves.

\section{Pure $[[17,11,3]]$-codes}

Let $k=6$. We work in $\mathbb{F}_{2}^{6}, Y$ consists of the vectors of weights 1 and 5 and the quantum code is defined by a family of 17 lines partitioning the points of weights different from 1 and 5 . One such partition is closely related to the $S_{6}$-generalized quadrangle. Write points of $\mathbb{F}_{2}^{6}$ in terms of subsets of $\{1,2,3,4,5,6\}$ such that 111000 for example is represented by 123 . Our partial spread consists of

- lines $l_{1}, \ldots l_{5}$ partitioning the points of weight 4 ;

- $l_{6}=\{123,456,123456\}$;

- $l_{7}=\{12,13,23\}, l_{8}=\{45,46,56\}$;

- 9 lines containing one point of weight 2 and two of weight 3 . Consider the permutations $\lambda, \rho$ defined by $\lambda=(1,2,3), \rho=(4,5,6)$. Let 


The union of two hyperovals on two planes of $\mathrm{PG}(3,4)$ intersecting in an exterior line clearly is a quantum cap $[[12,4,4]]$.

\section{References}

[1] J. Bierbrauer, Introduction to Coding Theory, Chapman and Hall, CRC Press, 2004.

full screen

close

[2] J. Bierbrauer and Y. Edel, Quantum twisted codes, J. Combin. Des. 8 (2000), 174-188.

[3] J. Bierbrauer, G. Faina, S. Marcugini and F. Pambianco, Additive quaternary codes of small length, Proceedings ACCT, Zvenigorod (Russia) September 2006, 15-18.

[4] J. Bierbrauer, Y. Edel, G. Faina, S. Marcugini and F. Pambianco, Short additive quaternary codes, IEEE Trans. Inform. Theory, to appear.

[5] A. Blokhuis and A. E. Brouwer, Small additive quaternary codes, European J. Combin. 25 (2004), 161-167.

[6] A. R. Calderbank, E. M. Rains, P. M. Shor and N. J. A. Sloane, Quantum error-correction via codes over GF(4), IEEE Trans. Inform. Theory 44 (1998), 1369-1387.

[7] C. Carlet, P. Charpin and V. Zinoviev, Codes, bent functions and permutations suitable for DES-like cryptosystems, Des. Codes Cryptogr. 15 (1998), 125-156.

[8] Y. Edel, http://www.mathi.uni-heidelberg.de/ yves.

[9] Y. Edel and J. Bierbrauer, 41 is the largest size of a cap in PG $(4,4)$, Des. Codes Cryptogr. 16(1999), 151-160.

[10] _ Large caps in small spaces, Des. Codes Cryptogr. 23 (2001), 197212.

[11] _ The largest cap in AG $(4,4)$ and its uniqueness, Des. Codes Cryptogr. 29 (2003), 99-104.

[12] D. Glynn, A 126-cap in $\mathrm{PG}(5,4)$ and its corresponding [126, 6, 88]-code, Util. Math. 55 (1999), 201-210.

[13] M. Grassl, http://www. codetables.de. 
Jürgen Bierbrauer

Department of Mathematical Sciences, Michigan Technological University, Houghton, MichigAN 49931, USA

e-mail: jbierbra@mtu.edu

Giorgio Faina

Dipartimento di Matematica e Informatica, Università degli Studi di Perugia, Italy e-mail: faina@dipmat.unipg.it

full screen

close

quit

NWWIII

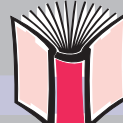

IIIIII

UNIVERSITEIT
Massimo Giulietti

Dipartimento di Matematica e Informatica, Università degli Studi di Perugia, Italy e-mail: giuliet@dipmat.unipg.it

\section{Stefano Marcugini}

Dipartimento di Matematica e Informatica, Università degli Studi di Perugia, Italy e-mail: gino@dipmat.unipg.it

\section{Fernanda Pambianco}

Dipartimento di Matematica e Informatica, Università degli Studi di Perugia, Italy e-mail: fernanda@dipmat.unipg.it 